\title{
Sevoflurane but not propofol enhances ovarian cancer cell malignancy through regulating cellular metabolic and signalling mechanisms
}

\author{
Cong $\mathrm{Hu}^{1}$, Bincheng Wang ${ }^{2}$, Zhigang $\mathrm{Liu}^{2}$, Qiling Chen ${ }^{2}$, Masashi Ishikawa ${ }^{2}$, Han Lin ${ }^{1}$, \\ Qingquan $\mathrm{Lian}^{1}$, jun $\mathrm{li}^{1}$, Jia $\mathrm{Li}^{2}$, and Daqing $\mathrm{Ma}^{2}$ \\ ${ }^{1}$ Wenzhou Medical University Second Affiliated Hospital \\ ${ }^{2}$ Imperial College London
}

February 25, 2021

\begin{abstract}
Background and Purpose: Surgery remains the first-line treatment of ovarian cancer. However, perioperative risk factors including the choice of anaesthetics may influence its recurrence after surgery. In the current study, it was hypothesised that inhalational anaesthetic sevoflurane and intravenous anaesthetic propofol might affect cancer cellular metabolism and signalling, which might interfere the malignancy of ovarian cancer cells. Experimental Approach: Cultured ovarian cancer cells were exposed to $2.5 \%$ sevoflurane or administered with $4 \mu \mathrm{g} / \mathrm{mL}$ propofol for 2 hours followed by 24 hours recovery. Their cell viability, proliferation, migration and invasion were assessed using cell counting kit-8, Ki-67 staining, wound healing and Transwell assay. Cellular signalling biomarkers were measured using immunofluorescent staining and/or Western blot. Cultured media were collected for 1H-NMR spectroscopy-based metabolomics analysis. Key Results: The cell viability, proliferation, migration, and invasion of ovarian cancer cells were enhanced by sevoflurane but suppressed by propofol. Sevoflurane increased the GLUT1, MPC1, GLUD1, p-Erk1/2, and HIF-1 $\alpha$ expressions but decreased the PEDF expression. In contrast to the sevoflurane treatment, the "mirror changes" of these cellular markers were observed with propofol. Sevoflurane increased levels of isopropanol but decreased glucose and glutamine levels in the media, but the opposite changes of those metabolites were found after propofol treatment. Conclusion and Implications: These data indicated that unlike propofol, sevoflurane enhanced ovarian cancer cell metabolism and activated PEDF/Erk/HIF-1 $\alpha$ cellular signalling pathway, suggesting that sevoflurane might have pro-tumour property but propofol might afford an anti-tumour property. The translational value of this work warrants further study.
\end{abstract}

\section{Introduction}

Ovarian cancer is the sixth most frequently diagnosed cancer and the seventh-highest mortality globally (Parkin, Bray, Ferlay, \& Pisani, 2005). There were an estimated more than 295,000 new cases and more than 184,000 deaths of ovarian cancer worldwide in 2018 (Bray et al., 2018). The treatments for ovarian cancer include surgery, chemotherapy, and radiotherapy. Among them, surgery remains the frontline therapy for ovarian cancer patients, as the tumour resection at the early stage can be very effective (Wyld, Audisio, \& Poston, 2015). However, ovarian cancer recurrence following surgery is still a leading cause of death (Saphner, Tormey, \& Gray, 1996; Zhu et al., 2011).

Many risk factors affect cancer recurrence, and one of them may be the choice of anaesthetics and anaesthesia techniques (Horowitz, Neeman, Sharon, \& Ben-Eliyahu, 2015). In an in vitro study, oestrogen receptorpositive or negative breast cancer cells were exposed to sevoflurane, and the proliferation and migration of both types of cancer cells were increased by sevoflurane (Ecimovic, McHugh, Murray, Doran, \& Buggy, 2013). An previous publication from our laboratory demonstrated that sevoflurane increased the migration 
of ovarian cancer cellsvia upregulating VEGFA, MMP11, CXCR2, and TГФ- $\beta$ gene expressions (Iwasaki et al., 2016). In contrary, propofol was reported to have an "anti-tumour" property; for example, our previous study demonstrated that propofol inhibited the proliferation and migration of prostate cancer cells (Huang et al., 2014). Clinically, a retrospective study reported that patients, who had surgery for the breast, colonic or rectal cancer, were under propofol-based total intravenous anaesthesia or sevoflurane inhalational anaesthesia and their overall one-year and five-year survival rates were higher with propofol anaesthesia (Enlund et al., 2014). However, the underlying mechanisms are unknown.

Glucose is taken up by cancer cells through glucose transporter 1 (GLUT1) and transformed to pyruvate, which is converted to lactate rather than enters mitochondria via mitochondrial pyruvate carrier 1 (MPC1) to be utilised by TCA cycle (Pezzuto, D'Ascanio, Ricci, Pagliuca, \& Carico, 2020; Zou et al., 2019). Glutamine can be transformed to be glutamate under the catalysation of glutamate dehydrogenase 1 (GLUD1) for using in the TCA cycle which generates crucial intermediates for cancer growth and survival (Craze et al., 2019). Such process is named as glutaminolysis for which GLUD1 is the key enzyme that locates in the inner membrane of mitochondria. Any factors that affect the GLUD1 expression and activity may interfere cancer cell survival (Son et al., 2013).

The transcriptional factor hypoxia-inducible factor-1 alpha (HIF-1 $\alpha$ ) plays a key role of the development of many tumour types, including breast, colonic and lung cancer (Zhao, Iwasaki, Yang, Savage, \& Ma, 2014) whilst the cellular signalling Erk1/2 pathway can regulate HIF-1 $\alpha$ (Karagiota, Kourti, Simos, \& Mylonis, 2019). Several regulators can regulate the signalling pathway of Erk1/2 and one of these regulators is the pigment epithelium-derived factor (PEDF), a member of the serine protease inhibitor family. The therapeutic values of PEDF were found for choroidal neovascularisation, heart disease and cancer (Filleur, Nelius, de Riese, \& Kennedy, 2009). Besides the upstream signalling of HIF-1 $\alpha$, the downstream effectors of HIF- $1 \alpha$ might also involve in the ovarian cancer malignancy. C-X-C motif chemokine 12 (CXCL12), also called stromal cell-derived factor 1 (SDF1), is originally correlated with the activity of leukocytes and its receptor is C-X-C chemokine receptor type 4 (CXCR4), also named as fusin or cluster of differentiation 184 (CD184) (Takano et al., 2014). It was found that CXCL12-CXCR4 was overexpressed in a variety of cancer types, and is involved in the progression of tumours (Scala et al., 2020).

In the current study, we hypothesised that unlike propofol, sevoflurane might upregulate the expressions of GLUT1, MPC1, and GLUD1, which increases the metabolism of ovarian cancer cells. The increased uptake of glucose inhibits the expression of PEDF and, in turn, upregulates p-Erk1/2, HIF-1 $\alpha$, CXCL12, and CXCR4. The alterations of metabolism and molecular entities by anaesthetics such as sevoflurane and propofol might change the malignancy of ovarian cancer cells.

\section{Methods}

Cell culture

Ovarian cancer cell line (SKOV3) was purchased from ECACC (Wiltshire, UK). SKOV3 cells were cultured in Gibco RPMI media 1640 (ThermoFisher, Paisley, UK) supplemented with $10 \%$ foetal bovine serum (FBS) and $1 \%$ penicillin-streptomycin (ThermoFisher, Paisley, UK) at $37^{\circ} \mathrm{C}$ with $5 \% \mathrm{CO}_{2}$ and balanced with air. SKOV3 cells were administered with $2.5 \%$ sevoflurane (ABOTT, Sittingbourne, UK), $4 \mu \mathrm{g} / \mathrm{mL}$ propofol (Sigma-Aldrich, Dorset, UK), media (naïve control), or intralipid (vehicle control) (Santa Cruz Biotechnology, Dallas, Texas, USA) for 2 hours, and followed by 24 hours recovery in fresh media.

Cell viability was assessed with CCK-8 assay

After recovery, SKOV3 cells were incubated with media plus cell counting kit-8 (CCK-8) solution at $37^{\circ} \mathrm{C}$ for 4 hours. The OD values of cancer cells were detected at a wavelength of $450 \mathrm{~nm}$. CCK- 8 solution with culture media but without cancer cells was used as blank. Cell viability values equalled to [OD (treatment) - OD (blank)] / [OD (control) - OD (blank)] X 100\%.

Cell proliferation was assessed with Ki-67 staining 
After recovery, SKOV3 cells were washed with phosphate-buffered saline (PBS) (Santa Cruz Biotechnology, Dallas, Texas, USA) and incubated in $4 \%$ paraformaldehyde (Santa Cruz Biotechnology, Dallas, Texas, USA) for 15 minutes. Cancer cells were washed with PBS plus 0.02\% Triton X-100 (Sigma-Aldrich, Dorset, UK) 3 times for 5 minutes each and blocked with 10\% donkey serum (Sigma-Aldrich, Dorset, UK) for 30 minutes. After blocking, cells were incubated with anti-Ki-67 antibody (1:200, rabbit polyclonal) (Santa Cruz Biotechnology, Dallas, Texas, USA) at $4^{\circ} \mathrm{C}$ overnight and anti-rabbit antibody (1:1000, goat polyclonal) (Abcam, Cambridge, UK) for 1 hour at room temperature under dark in sequence. The 4',6-diamidino-2phenylindole (DAPI) mounting media (Vector Laboratories, Burlingame, California, USA) were mounted on cancer cells. The fluorescent images were taken under fluorescence microscope and the percentage of Ki-67 positive cells was calculated by Fiji (ImageJ 2.0) software (National Institutes of Health, Bethesda, Maryland, USA).

Cell migration was assessed with wound healing assay

SKOV3 cells were seeded in petri dishes to form a continuous monolayer. Cancer cells were incubated in pure media, $2.5 \%$ sevoflurane, or $4 \mu \mathrm{g} / \mathrm{mL}$ propofol for 2 hours. The monolayer of cancer cells was scratched to form a cell-free gap. The petri dishes were washed with PBS to discard suspended cancer cells. The cell-free gap was captured under a microscope as the baseline. After incubated with culture media for 24 hours, the cell-free gap was captured again under the microscope to calculate the migrate capability of cancer cells with Fiji (ImageJ 2.0) software (National Institutes of Health, Bethesda, Maryland, USA).

Cell invasion was assessed with Transwell assay

SKOV 3 cells were seeded in petri dishes and administered with media, $2.5 \%$ sevoflurane, or $4 \mu \mathrm{g} / \mathrm{mL}$ propofol for 2 hours. After administration, cancer cells were mixed with FBS-free media and seeded in the upper chamber of the Transwell assay kit, which was pre-embedded with Corning Matrigel matrix (Corning, New York, USA). The FBS-enriched media was placed into the lower chamber of the Transwell assay kit. After incubated at $37^{\circ} \mathrm{C}$ for 24 hours, the upper chamber was inserted in the $70 \%$ methanol (ThermoFisher, Paisley, UK) for 30 minutes. The cancer cells on the upper chamber were dyed with $0.1 \%$ crystal violet (Sigma-Aldrich, Dorset, UK) for 15 minutes and washed to get away the leftover dye. The cells on the upper membrane of the upper chamber were removed. The cells on the bottom membrane of it were regarded as invasive cells and detected by a microscope. The number of invasive cells was counted with Fiji (ImageJ 2.0) software (National Institutes of Health, Bethesda, Maryland, USA).

GLUT1, MPC1, PEDF, HIF-1 $\alpha$, CXCL12, and CXCR4 expressions were detected with immunofluorescent staining

After recovery, SKOV3 cells were washed with phosphate-buffered saline (PBS) (Santa Cruz Biotechnology, Dallas, Texas, USA) and incubated in 4\% paraformaldehyde (Santa Cruz Biotechnology, Dallas, Texas, USA) for 15 minutes. Cells were washed with PBS plus 0.02\% Triton X-100 (Sigma-Aldrich, Dorset, UK) 3 times for 5 minutes each and blocked with $10 \%$ donkey serum (Sigma-Aldrich, Dorset, UK) for 30 minutes. After blocking, cells were incubated with primary antibody at $4^{\circ} \mathrm{C}$ overnight and secondary antibody for 1 hour at room temperature under dark in sequence (Table S1 ). The 4',6-diamidino-2-phenylindole (DAPI) mounting media (Vector Laboratories, Burlingame, California, USA) were mounted on cancer cells. The expressions of proteins were detected by a fluorescence microscope.

GLUT1, MPC1, GLUD1, PEDF, p-Erk1/2, Erk1/2, and HIF-1 $\alpha$ expressions were determined with Western blot analysis

After recovery, SKOV3 cells were adapted with cell lysis buffer (Cell signalling Technology, London, UK) to extract proteins. The protein samples were mixed with NuPAGE LDS sample buffer (ThermoFisher, Paisley, UK) and boiled at $95^{\circ} \mathrm{C}$ for 10 minutes. The boiled samples were loaded onto a NuPAGE BisTris gel (ThermoFisher, Paisley, UK) for electrophoresis. After electrophoresis, the protein on the gel was transferred to a nitrocellulose membrane (ThermoFisher, Paisley, UK). The membrane was blocked with $5 \%$ non-fat milk for 1 hour and incubated with primary antibody at $4^{\circ} \mathrm{C}$ overnight and secondary antibody 
on the next day (Table S1 ) for 1 hour and adapted with luminol reagent solution A and B (Santa Cruz Biotechnology, Dallas, Texas, USA). The protein bands were detected by GeneSnap Version 7.1 (Syngene, Cambridge, UK) and the levels of protein were analysed by Fiji (ImageJ 2.0) software (National Institutes of Health, Bethesda, Maryland, USA).

The metabolic alterations in media were measured with proton NMR spectroscopy

After recovery, the culture media of SKOV3 cells were collected. One day before the NMR experiment, media samples were thawed at the room temperature. The sample with $540 \mu \mathrm{L}$ was collected and mixed with 60 $\mu \mathrm{L}$ potassium phosphate buffer $(\mathrm{pH}=7.4)$ containing $1.5 \mathrm{M} \mathrm{KH}_{2} \mathrm{PO}_{4}, 1 \mathrm{mM} \mathrm{NaN}_{3}, 0.1 \%$ TSP and $\mathrm{D}_{2} \mathrm{O}$. A total of $580 \mu \mathrm{L}$ mixture was placed into an NMR tube (Bruker Corporation, Rheinstetten, Germany) with an outer diameter of $5 \mathrm{~mm}$. ${ }^{1} \mathrm{H}-\mathrm{NMR}$ spectra of media samples were obtained using a Bruker $600 \mathrm{MHz}$ spectrometer (Bruker Corporation, Rheinstetten, Germany) at the operating ${ }^{1} \mathrm{H}$ frequency of $600.13 \mathrm{MHz}$ at a temperature of $300 \mathrm{~K}$. A standard NMR pulse sequence (recycle delay- $90^{\circ}-t_{1}-90^{0}-t_{m}-90^{\circ}$ acquisition) was applied to acquire ${ }^{1} \mathrm{H}-\mathrm{NMR}$ spectral data $\left(\mathrm{t}_{1}=3 \mu \mathrm{s}, \mathrm{t}_{\mathrm{m}}=100 \mathrm{~ms}\right)$. The water peak suppression was achieved using selective irradiation during a recycle delay of $4 \mathrm{~s}$ and $\mathrm{t}_{\mathrm{m}}$. A $90^{\circ}$ pulse was adjusted to $\sim 10 \mu \mathrm{s}$. A total of 32 scans for cell media were collected into $64 \mathrm{k}$ data points with a spectral width of $20 \mathrm{ppm} .{ }^{1} \mathrm{H}-\mathrm{NMR}$ spectral data were acquired using TopSpin 4.0.9 (Bruker Corporation, Rheinstetten, Germany). The spectral data were imported into MATLAB R2018a (MathWorks, Natick, Massachusetts, USA) and SIMCA (Sartorius, Gottingen, Germany) for multivariate statistical analysis. The chemical shift ranged from 4.7 to 5.0, and from -1 to 0.3 was cut. The spectral data was aligned with recursive segment-wise peak alignment method and normalised with probabilistic quotient normalisation method (Dieterle, Ross, Schlotterbeck, \& Senn, 2006; Veselkov et al., 2009). Metabolites were identified using Chenomx software (CHENOMX, Edmonton, Canada).

\section{Materials}

The SKOV3 cell line was purchased from ECACC, Wiltshire, UK. The Gibco RPMI media 1640, penicillinstreptomycin, methanol, NuPAGE LDS sample buffer, NuPAGE Bis-Tris gel and nitrocellulose membrane were obrained from ThermoFisher, Paisley, UK. The sevoflurane was purchased from ABOTT, Sittingbourne, UK. The propofol, Triton X-100, donkey serum and crystal violet were acquired from Sigma-Aldrich, Dorset, UK. The intralipid, PBS, paraformaldehyde, anti-Ki-67 antibody and luminol reagent solution were from Santa Cruz Biotechnology, Dallas, Texas, USA. The anti-rabbit and anti-mouse antibodies for immunofluorescent staining, anti-GLUT1, anti-GLUD1, anti-PEDF, anti-CXCL12 and anti-CXCR4 antibodies were obtained from Abcam, Cambridge, UK. The DAPI mounting media was acquired from Vector Laboratories, Burlingame, California, USA. The Corning Matrigel matrix was purchased from Corning, New York, USA. The anti-MPC1 and anti-HIF-1 $\alpha$ antibodies were obtained from Novus Biologicals, Abingdon, UK. The antip-Erk1/2 and anti-Erk1/2 antibodies, anti-rabbit and anti-mouse antibodies for Western blot analysis, and cell lysis buffer were acquired from Cell signalling Technology, London, UK. The anti-GAPDH antibody was purchased from Merck, Hertfordshire, UK. The NMR tube was from Bruker Corporation, Rheinstetten, Germany.

\section{Results}

Effects of sevoflurane or propofol on cancer malignancy in ovarian cancer cells

Sevoflurane significantly increased the ovarian cancer cell viability of compared to naïve control (NC vs . S, $100.0 \pm 1.5$ vs $.122 .0 \pm 4.1, \mathrm{p}<0.0001, \mathrm{n}=6$ ), while propofol significantly decreased the cell viability (NC vs . S, $100.0 \pm 1.5$ vs . $61.6 \pm 5.4, \mathrm{p}<0.0001, \mathrm{n}=6)($ Fig $\mathbf{1 A})$. The proliferation of SKOV3 cells was indicated by the number of Ki-67 positive cells (Fig 1B ). It was found that the number of Ki-67 positive cells was significantly increased by sevoflurane administration ( $\mathrm{NC}$ vs . S, $32.0 \pm 5.1$ vs $.52 .4 \pm 13.8, \mathrm{p}<$ $0.01, \mathrm{n}=6$ ). However, Ki-67 positive cells were significantly decreased by propofol treatment (NC vs . P, $32.0 \pm 5.1$ vs $.16 .0 \pm 7.3, \mathrm{p}<0.05, \mathrm{n}=6)($ Fig $\mathbf{1 C})$.

The migrate capability of ovarian cancer cells after sevoflurane treatment was significantly greater than that 
of the naïve control ( $\mathrm{NCvs}$. S, $55.2 \pm 7.5$ vs . $92.5 \pm 8.2, \mathrm{p}<0.0001, \mathrm{n}=6$ ) (Fig 1D ). In contrast, the migrate capability of cancer cells was inhibited by propofol exposure ( $\mathrm{NC}$ vs . $\mathrm{P}, 55.2 \pm 7.5 \mathrm{vs} .34 .3$ $\pm 9.4, \mathrm{p}<0.01, \mathrm{n}=6)(\mathbf{F i g} \mathbf{1 E})$. The invasion of ovarian cancer cells was evaluated by comparing the number of invasive cells with Transwell assay. Significantly higher number of invasive cells was observed in the sevoflurane group compared to naïve control (NC vs . S, $1.0 \pm 0.1$ vs $.2 .2 \pm 0.6, \mathrm{p}<0.0001, \mathrm{n}=6$ ) (Fig 1F ), whereas propofol exhibited an opposite effect ( $\mathrm{NC}$ vs . P, $1.0 \pm 0.1$ vs $0.4 \pm 0.1, \mathrm{p}<0.05, \mathrm{n}=$ 6) (Fig 1G).

Effects of sevoflurane or propofol on the expressions of GLUT1, MPC1, and GLUD1 in ovarian cancer cells

Based on the immunofluorescent staining of cells, the intensities of GLUT1 (Fig 2A ) and MPC1 (Fig 2B ) were higher in the sevoflurane group compared to naïve control, but much lower in the propofol group than the naïve control. From Western blot analysis, the protein expression levels of GLUT1 (NC vs . S, $1.0 \pm$ 0.1 vs . $1.4 \pm 0.3, \mathrm{p}<0.01, \mathrm{n}=6$ ) and MPC1 ( $\mathrm{NC}$ vs . S, $1.0 \pm 0.3$ vs $.1 .5 \pm 0.2, \mathrm{p}<0.01, \mathrm{n}=6$ ) were significantly increased after sevoflurane exposure (Fig 2C ). However, the expression levels of GLUT1 (NC vs . $\mathrm{P}, 1.0 \pm 0.1$ vs . $0.3 \pm 0.1, \mathrm{p}<0.0001, \mathrm{n}=6$ ) and MPC1 (NCvs . $\mathrm{P}, 1.0 \pm 0.3$ vs . $0.6 \pm 0.3, \mathrm{p}<0.05$, $\mathrm{n}=6$ ) were significantly decreased after propofol treatment compared to the naïve control (Fig $\mathbf{2 E}$ and F ). Similarly, the expression level of glutamate dehydrogenase 1 (GLUD1) was significantly increased by sevoflurane administration ( NC vs . S, $1.0 \pm 0.1$ vs . $1.4 \pm 0.1, \mathrm{p}<0.01, \mathrm{n}=6$ ), but decreased by propofol administration ( $\mathrm{NC}$ vs . P, $1.0 \pm 0.1$ vs . $0.6 \pm 0.2, \mathrm{p}<0.001, \mathrm{n}=6)($ Fig $2 \mathrm{D})$.

Effects of sevoflurane or propofol on the cellular metabolism of SKOV3 cells

The metabolic profiles obtained from media samples of naïve control, vehicle control, sevoflurane, and propofol groups were analysed using unsupervised PCA analysis with 2 principal components. The PCA scores plot showed a clear grouping pattern among these four groups (Fig 3A ). Pair-wise comparisons of naïve control vs . sevoflurane group and vehicle control vs . propofol group were carried out using OPLS-DA analysis with one predictive component and one orthogonal component. Permutation $\mathrm{p}$ values showed both models of naïve control $v s$. sevoflurane $(\mathrm{p}=0.005)$ and vehicle controlvs . propofol $(\mathrm{p}=0.005)$ had significances. The permutation $p$ values together with $\mathrm{R}^{2} \mathrm{X}, \mathrm{Q}^{2} \mathrm{X}$, and $\mathrm{Q}^{2} \mathrm{Y}$ of two models were summarised (Table 1 ). The OPLS-DA scores plots showed there were clear separations between naïve control and sevoflurane group (Fig 3B ), and vehicle control and propofol group (Fig 3D ). The OPLS-DA loadings plots showed that the concentrations of glucose and glutamine were decreased, and the concentration of isopropanol was increased in the sevoflurane group compared to the naïve control group (Fig 3C ). However, the concentrations of glucose and glutamine were increased, and the concentration of isopropanol was decreased in the propofol group in contrast to vehicle control group (Fig 3E ). After anaesthetics administration, the concentrations of lactate, pyruvate, acetate, alanine, valine, and leucine were increased by both sevoflurane and propofol. Furthermore, propofol also increased the levels of glycerol, fatty acids, asparagine, succinate, acetone, arginine, and isoleucine, but decreased the level of ethanol (Table 2 ).

Regulations of sevoflurane or propofol on PEDF expression, Erk pathway, and HIF-1 $\alpha$ expression

From immunofluorescent staining, it was found that the fluorescent intensity of pigment epithelium-derived factor (PEDF) was decreased in the sevoflurane group but increased in the propofol group compared to naïve control (Fig 4A ). The result was validated using Western blot analysis, which showed the significantly lower expression level of PEDF after sevoflurane exposure ( $\mathrm{NC}$ vs . S, $1.0 \pm 0.1$ vs $.0 .6 \pm 0.2, \mathrm{p}<0.0001, \mathrm{n}=$ 6 ) but significantly higher after propofol exposure ( $\mathrm{NC}$ vs . $\mathrm{P}, 1.0 \pm 0.1$ vs $.1 .2 \pm 0.1, \mathrm{p}<0.05, \mathrm{n}=6$ ) (Fig 4D ). Different from PEDF expression levels, the Western blot analysis showed that the expression level ratio of p-Erk1/2 to Erk1/2 was significantly increased in the sevoflurane group (NC vs . S, $1.0 \pm 0.1$ vs . $1.3 \pm 0.2, \mathrm{p}<0.05, \mathrm{n}=6$ ), but decreased in the propofol group compared to the control ( $\mathrm{NC}$ vs . P, $1.0 \pm 0.1$ vs $.0 .7 \pm 0.2, \mathrm{p}<0.05, \mathrm{n}=6)($ Fig $4 \mathbf{E})$. Similarly, the fluorescent staining showed SKOV3 cells administered with sevoflurane had a higher intensity of hypoxia-inducible factor 1-alpha (HIF-1 $\alpha$ ) than the naïve control, while those of the propofol group had a lower intensity of HIF-1 $\alpha$ than control (Fig 4B ). From Western blot analysis, it was also found that the expression level of HIF- $1 \alpha$ was significantly increased 
after cancer cells administered with sevoflurane (NCvs . S, $1.0 \pm 0.3$ vs . $1.5 \pm 0.5, \mathrm{p}<0.05, \mathrm{n}=6$ ), but significantly decreased after propofol treatment compared to the control (NC vs. S, $1.0 \pm 0.3$ vs. $0.4 \pm 0.3$, $\mathrm{p}<0.05, \mathrm{n}=6)(\mathbf{F i g} \mathbf{4 F})$.

Effects of sevoflurane or propofol on CXCL12 and CXCR4 expressions

According to immunofluorescent staining, the intensity of C-X-C motif chemokine ligand 12 (CXCL12) was increased in the sevoflurane group but decreased in the propofol group (Fig 5A ). In similar, the fluorescent staining of C-X-C motif chemokine receptor 4 (CXCR4) was higher in the sevoflurane group than the control, and the intensity of the marker was lower in the propofol group than the control (Fig 5B ). These results showed the expressions of CXCL12 and CXCR4 were both increased by sevoflurane but decreased by propofol administration.

\section{Discussion}

Our current study suggested that inhalational anaesthetic sevoflurane enhanced ovarian cancer cell viability, proliferation, migration, and invasion. In contrast, intravenous anaesthetic propofol inhibited those cellular activities. These phenotypic observations could be associated with upregulated expressions of GLUT1, MPC1, GLUD1, p-Erk1/2, HIF-1 $\alpha$, CXCL12 and CXCR4, and downregulated PEDF expression in the sevoflurane group. Sevoflurane, but not propofol, promoted metabolism of ovarian cancer cells by enhancing the uptake of the metabolic substrates such as glucose and glutamine.

The lower concentration of glucose and higher concentration of pyruvate in the media were found after sevoflurane exposure compared to the naïve control, which were in agreement with observed upregulated expression level of GLUT1. The enhanced activity of GLUT1 may transport more glucose into the cytoplasm to convert to pyruvate, which explained the metabolic changes in the media after sevoflurane administration. In contrast, propofol downregulated GLUT1, which resulted in the less uptake of glucose from media, and hence the concentration of glucose was increased after propofol exposure. The cellular glucose uptakevia high level of GLUT1 expression was correlated with the malignancy of cancers (Leung, 2004; Pezzuto et al., 2020) and the overexpression of GLUT1 in cancer cells were essential for the high rate of glycolysis (Wright, 2020). Propofol was reported to downregulateGLUT1 gene in macrophages (Tanaka et al., 2010) and this was in line with the downregulation of GLUT1 proteins in the rat brain tissue under hypoxic preconditioning (Xiao et al., 2020). This is quite similar to cancer cell scenario, as, under high proliferative rate, cancer cells had inadequate oxygen supply and were actually under hypoxic condition (Mudassar, Shen, O'Neill, \& Hau, 2020). These supported our findings that the malignancy of ovarian cancer cells was related to the expression of GLUT1 after anaesthetic administration.

The concentration of lactate was increased in both sevoflurane and propofol treatments, which might be resulted from the increased pyruvate in glycolysis and then generated more lactate. It was reported that sevoflurane and propofol both increased the lactate level in the blood of dogs (Söbbeler et al., 2018). Another study in mice also demonstrated that sevoflurane increased pyruvate and lactate levels (Horn \& Klein, 2010). All these reports were in line with the findings of our current study.

It was found that both the expressions of MPC1 and GLUD1 were upregulated after sevoflurane exposure but were downregulated after propofol treatment. Besides, the concentration of glutamine in media was decreased after sevoflurane administration. MPC1, a member of mitochondrial carrier system, locates at the inner membrane of mitochondria and transports pyruvate into mitochondria from cytoplasm (Taylor, 2017). The expression of MPC1 is decreased in most tumour types especially those under a high rate of proliferation as related to the increased rate of glycolysis. This suggests that pyruvate likely shifts from the mitochondrial TCA cycle to cytoplasm glycolysis, which does not require oxygen supply as cancer cells are usually under hypoxic condition (Rauckhorst \& Taylor, 2016). In addition, glutaminolysis compensates for the disturbed function of the TCA cycle due to less pyruvate intake and cancer cells, in turn, uptake glutamine and convert them into glutamate under the activation of GLUD1. The glutamate can be used by TCA cycle to restore the survival of cancer cells as the intermediates of TCA cycle are the source for synthesis of amino acids, proteins, fatty acids, lipids, carbon skeleton, and nucleic acids (Yoo, Yu, Sung, \& 
Han, 2020). Evidence from other studies showed propofol might disturb the mitochondrial respiratory chain, which was related to TCA cycle (Berndt et al., 2018). It was also reported that unlike inhibitory effects of propofol, sevoflurane preserved the function of the mitochondrial respiratory chain in a myocardial ischaemic model (Lotz, Stumpner, \& Smul, 2020). Our data demonstrated that after sevoflurane administration, the MPC1 and GLUD1 expressions were upregulated, which might enhance the activity of the TCA cycle to meet the demands of cancer survival and progression. The decreased concentration of glutamine in media suggested that the utilisation of glutamine was likely increased and glutaminolysis was then promoted. With the disturbed function of the TCA cycle after propofol exposure, amino acids that can be used in the TCA cycle were accumulated, such as asparagine and arginine (Pasini et al., 2018), which was consistent with our findings.

Except glucose and glutamine, another "mirror change" of metabolites between sevoflurane and propofol administration was isopropanol. It was reported that the level of isopropanol was increased in the exhaled breath of lung cancer patients, and it had been regarded as a potential biomarker for lung cancer diagnosis (Chien et al., 2017). It seemed the level of isopropanol had some correlations with cancer malignancy, which was consistent with the findings of this study that sevoflurane enhanced the malignancy of ovarian cancer cells and increased the level of isopropanol, while propofol inhibited the malignancy of ovarian cancer cells and decreased the level of isopropanol. Isopropanol can be reversibly converted to acetone (Beauchamp, Valento, \& Kim, 2016; Li, Liu, Liu, Cheng, \& Duan, 2017), which may also contribute to the increased level of acetone in the media of the propofol group.

The levels of glycerol and fatty acids were increased in the propofol group. Through $\beta$-oxidation of fatty acids, acetyl-CoA is generated and used in the TCA cycle (Y. Liu, 2006). Thus, the changes of glycerol and fatty acids in the propofol group were another evidence that the mitochondria function and TCA cycle was inhibited or disturbed by propofol treatment. The glycerol and fatty acids might also come from cell membrane degradation and phospholipids broke down into them. From an earlier study, it was found that propofol affected the membrane ultrastructure of HeLa cells that the surface roughness of cellular membrane was decreased in a dose-dependent manner (Zhang et al., 2016).

In the current study, the expression level of PEDF was decreased after sevoflurane administration but increased after propofol treatment. In human retinal pigment epithelium, the expression of GLUT1 was increased under hypoxia condition that resulted in the increased uptake of glucose, which led to a decrease of PEDF expression (Calado, Alves, Simão, \& Silva, 2016). Another study also reported that the overexpression of the PEDF gene in mice was related to the reduction of glucose uptake and decreased expression of GLUT1 (Calado, Diaz-Corrales, \& Silva, 2016). These reports were consistent with our findings that sevoflurane increased the GLUT1 expression and glucose uptake, which led to a downregulated expression of PEDF. However, an opposite effect was found with propofol treatment.

In the current study, Erk1/2 signalling pathway was induced after sevoflurane exposure but inhibited after propofol exposure. There was evidence that the increased expression of PEDF was related to the inhibition of Erk1/2 signalling pathway in a diabetic model (Dong et al., 2019), which was in line with our results. HIF- $1 \alpha$ is a transcriptional factor that can be regulated by a variety of signalling pathways, and Erk1/2 pathway is one of them (R. M. Liu, Xu, Chen, Feng, \& Xie, 2020). In cancer cells, the HIF-1 $\alpha$ is overexpressed, which regulates tumour survival-related genes, such as CXCL12 and CXCR4 (Gola et al., 2020; Xue et al., 2020). It was in line with the results of the current study that sevoflurane upregulated Erk1/2 signalling pathway, and HIF-1 $\alpha$, CXCL12 and CXCR4 expressions, while propofol downregulated these molecular entities.

Our study has some limitations. Firstly, the causal relationship between cellular signalling changes and metabolic alterations induced by anaesthetics remains unknown. However, it is very likely that, for example, sevoflurane promotes cancer cell survival and development due to survival cellular signalling pathway activation whereby more energy substrates use up for cell proliferation and growth. Secondly, our cultured cell study may not be relevant to human. Therefore, the implications of our current study may be limited. However, in some clinical studies, breast, colonic and rectal cancer patients were anaesthetised with inhalational anaesthetics sevoflurane or desflurane, or intravenous anaesthetic propofol during surgery and the survival 
rate of propofol anaesthetised patients were significantly higher than those with inhalational anaesthesia (Enlund et al., 2014; Wu et al., 2018). Laboratory data including the one reported here and retrospective clinical data all point to that sevoflurane might be a risk factor for cancer patients, while propofol may be beneficial to cancer patients for their surgery. Therefore, clinical studies are urgently needed to evaluate anaesthesia regimens for cancer patients to optimise the surgical outcomes.

Conclusions

The current study demonstrated that sevoflurane upregulated GLUT1, MPC1, and GLUD1 expressions of ovarian cancer cells, while propofol downregulated the expressions of these molecules. These regulations by sevoflurane or propofol on ovarian cancer cells led to different metabolic features. The upregulated GLUT1 expression and, in turn, increased glucose uptake after sevoflurane exposure resulted in a decreased expression of PEDF. In opposite, an increased expression of PEDF was identified after propofol treatment. Furthermore, in contrast to propofol, sevoflurane upregulated Erk1/2 pathway, HIF-1 $\alpha$, CXCL12 and CXCR4 expressions through PEDF inhibition per se. In summary, the profiling alterations of molecular and metabolic modulations found in the present study indicate the pro- and anti-tumour properties of sevoflurane and propofol, respectively. The translational value of these is subjected to study further in clinical settings.

\section{References}

Beauchamp, G. A., Valento, M., \& Kim, J. (2016). Toxic alcohol ingestion: prompt recognition and management in the emergency department [digest]. Emerg Med Pract, 18 (9 Suppl Points \& Pearls), S1-s2.

Berndt, N., Rösner, J., Haq, R. U., Kann, O., Kovács, R., Holzhütter, H. G., . . Liotta, A. (2018). Possible neurotoxicity of the anesthetic propofol: evidence for the inhibition of complex II of the respiratory chain in area CA3 of rat hippocampal slices. Arch Toxicol, 92 (10), 3191-3205. doi:10.1007/s00204-018-2295-8

Bray, F., Ferlay, J., Soerjomataram, I., Siegel, R. L., Torre, L. A., \& Jemal, A. (2018). Global cancer statistics 2018: GLOBOCAN estimates of incidence and mortality worldwide for 36 cancers in 185 countries. CA Cancer J Clin, 68 (6), 394-424. doi:10.3322/caac.21492

Calado, S. M., Alves, L. S., Simão, S., \& Silva, G. A. (2016). GLUT1 activity contributes to the impairment of PEDF secretion by the RPE.Mol Vis, 22, 761-770.

Calado, S. M., Diaz-Corrales, F., \& Silva, G. A. (2016). pEPito-driven PEDF Expression Ameliorates Diabetic Retinopathy Hallmarks. Hum Gene Ther Methods, 27 (2), 79-86. doi:10.1089/hgtb.2015.169

Chien, P. J., Suzuki, T., Tsujii, M., Ye, M., Toma, K., Arakawa, T., . . Mitsubayashi, K. (2017). Bio-sniffer (gas-phase biosensor) with secondary alcohol dehydrogenase (S-ADH) for determination of isopropanol in exhaled air as a potential volatile biomarker. Biosens Bioelectron, 91 , 341-346. doi:10.1016/j.bios.2016.12.050

Craze, M. L., El-Ansari, R., Aleskandarany, M. A., Cheng, K. W., Alfarsi, L., Masisi, B., . . Green, A. R. (2019). Glutamate dehydrogenase (GLUD1) expression in breast cancer. Breast Cancer Res Treat, 174 (1), 79-91. doi:10.1007/s10549-018-5060-z

Dieterle, F., Ross, A., Schlotterbeck, G., \& Senn, H. (2006). Probabilistic quotient normalization as robust method to account for dilution of complex biological mixtures. Application in 1H NMR metabonomics. Anal Chem, 78 (13), 4281-4290. doi:10.1021/ac051632c

Dong, Y., Wan, G., Yan, P., Qian, C., Li, F., \& Peng, G. (2019). Fabrication of resveratrol coated gold nanoparticles and investigation of their effect on diabetic retinopathy in streptozotocin induced diabetic rats. J Photochem Photobiol B, 195 , 51-57. doi:10.1016/j.jphotobiol.2019.04.012

Ecimovic, P., McHugh, B., Murray, D., Doran, P., \& Buggy, D. J. (2013). Effects of sevoflurane on breast cancer cell function in vitro.Anticancer Res, 33 (10), 4255-4260.

Enlund, M., Berglund, A., Andreasson, K., Cicek, C., Enlund, A., \& Bergkvist, L. (2014). The choice of anaesthetic-sevoflurane or propofol-and outcome from cancer surgery: a retrospective analysis. Ups J Med 
Sci, 119 (3), 251-261. doi:10.3109/03009734.2014.922649

Filleur, S., Nelius, T., de Riese, W., \& Kennedy, R. C. (2009). Characterization of PEDF: a multi-functional serpin family protein.J Cell Biochem, 106 (5), 769-775. doi:10.1002/jcb.22072

Gola, C., Iussich, S., Noury, S., Martano, M., Gattino, F., Morello, E., . . . De Maria, R. (2020). Clinical significance and in vitro cellular regulation of hypoxia mimicry on HIF- $1 \alpha$ and downstream genes in canine appendicular osteosarcoma. Vet J, 264 , 105538. doi:10.1016/j.tvj1.2020.105538

Horn, T., \& Klein, J. (2010). Lactate levels in the brain are elevated upon exposure to volatile anesthetics: a microdialysis study.Neurochem Int, 57 (8), 940-947. doi:10.1016/j.neuint.2010.09.014

Horowitz, M., Neeman, E., Sharon, E., \& Ben-Eliyahu, S. (2015). Exploiting the critical perioperative period to improve long-term cancer outcomes. Nat Rev Clin Oncol, 12 (4), 213-226. doi:10.1038/nrclinonc.2014.224

Huang, H., Benzonana, L. L., Zhao, H., Watts, H. R., Perry, N. J., Bevan, C., . . . Ma, D. (2014). Prostate cancer cell malignancy via modulation of HIF-1 $\alpha$ pathway with isoflurane and propofol alone and in combination. Br J Cancer, 111 (7), 1338-1349. doi:10.1038/bjc.2014.426

Iwasaki, M., Zhao, H., Jaffer, T., Unwith, S., Benzonana, L., Lian, Q., . . . Ma, D. (2016). Volatile anaesthetics enhance the metastasis related cellular signalling including CXCR2 of ovarian cancer cells. Oncotarget, 7 (18), 26042-26056. doi:10.18632/oncotarget.8304

Karagiota, A., Kourti, M., Simos, G., \& Mylonis, I. (2019). HIF-1 $\alpha$-derived cell-penetrating peptides inhibit ERK-dependent activation of HIF-1 and trigger apoptosis of cancer cells under hypoxia. Cell Mol Life Sci, 76 (4), 809-825. doi:10.1007/s00018-018-2985-7

Leung, K. (2004). 8-[(123)I]Iodo-L-1,2,3,4-tetrahydro-7-hydroxyisoquinoline-3-carboxylic acid. In Molecular Imaging and Contrast Agent Database (MICAD) . Bethesda (MD): National Center for Biotechnology Information (US).

Li, W. W., Liu, Y., Liu, Y., Cheng, S. Q., \& Duan, Y. X. (2017). Exhaled isopropanol: new potential biomarker in diabetic breathomics and its metabolic correlations with acetone. Rsc Advances, 7 (28), 17480-17488. doi:10.1039/c7ra00815e

Liu, R. M., Xu, P., Chen, Q., Feng, S. L., \& Xie, Y. (2020). A multiple-targets alkaloid nuciferine overcomes paclitaxel-induced drug resistance in vitro and in vivo. Phytomedicine, 79 , 153342. doi:10.1016/j.phymed.2020.153342

Liu, Y. (2006). Fatty acid oxidation is a dominant bioenergetic pathway in prostate cancer. Prostate Cancer Prostatic Dis, 9 (3), 230-234. doi:10.1038/sj.pcan.4500879

Lotz, C., Stumpner, J., \& Smul, T. M. (2020). Sevoflurane as opposed to propofol anesthesia preserves mitochondrial function and alleviates myocardial ischemia/reperfusion injury. Biomed Pharmacother, 129, 110417. doi:10.1016/j.biopha.2020.110417

Mudassar, F., Shen, H., O’Neill, G., \& Hau, E. (2020). Targeting tumor hypoxia and mitochondrial metabolism with anti-parasitic drugs to improve radiation response in high-grade gliomas. $J$ Exp Clin Cancer Res, 39 (1), 208. doi:10.1186/s13046-020-01724-6

Parkin, D. M., Bray, F., Ferlay, J., \& Pisani, P. (2005). Global cancer statistics, 2002. Ca-a Cancer Journal for Clinicians, 55 (2), 74-108. doi:10.3322/canjclin.55.2.74

Pasini, E., Corsetti, G., Aquilani, R., Romano, C., Picca, A., Calvani, R., \& Dioguardi, F. S. (2018). ProteinAmino Acid Metabolism Disarrangements: The Hidden Enemy of Chronic Age-Related Conditions.Nutrients, 10 (4). doi:10.3390/nu10040391

Pezzuto, A., D’Ascanio, M., Ricci, A., Pagliuca, A., \& Carico, E. (2020). Expression and role of p16 and GLUT1 in malignant diseases and lung cancer: A review. Thorac Cancer . doi:10.1111/1759-7714.13651 
Rauckhorst, A. J., \& Taylor, E. B. (2016). Mitochondrial pyruvate carrier function and cancer metabolism. Curr Opin Genet Dev, 38 , 102-109. doi:10.1016/j.gde.2016.05.003

Saphner, T., Tormey, D. C., \& Gray, R. (1996). Annual hazard rates of recurrence for breast cancer after primary therapy. J Clin Oncol, 14 (10), 2738-2746. doi:10.1200/jco.1996.14.10.2738

Scala, S., D’Alterio, C., Milanesi, S., Castagna, A., Carriero, R., Farina, F. M., . . . Borroni, E. M. (2020). New Insights on the Emerging Genomic Landscape of CXCR4 in Cancer: A Lesson from WHIM. Vaccines (Basel), 8 (2). doi:10.3390/vaccines8020164

Söbbeler, F. J., Carrera, I., Pasloske, K., Ranasinghe, M. G., Kircher, P., \& Kästner, S. B. R. (2018). Effects of isoflurane, sevoflurane, propofol and alfaxalone on brain metabolism in dogs assessed by proton magnetic resonance spectroscopy ((1)H MRS). BMC Vet Res, 14 (1), 69. doi:10.1186/s12917-018-1396-1

Son, J., Lyssiotis, C. A., Ying, H., Wang, X., Hua, S., Ligorio, M., . . Kimmelman, A. C. (2013). Glutamine supports pancreatic cancer growth through a KRAS-regulated metabolic pathway. Nature, 496 (7443), 101105. doi:10.1038/nature 12040

Takano, T., Li, Y. J., Kukita, A., Yamaza, T., Ayukawa, Y., Moriyama, K., . . Kukita, T. (2014). Mesenchymal stem cells markedly suppress inflammatory bone destruction in rats with adjuvant-induced arthritis. Lab Invest, 94 (3), 286-296. doi:10.1038/labinvest.2013.152

Tanaka, T., Takabuchi, S., Nishi, K., Oda, S., Wakamatsu, T., Daijo, H., . . Hirota, K. (2010). The intravenous anesthetic propofol inhibits lipopolysaccharide-induced hypoxia-inducible factor 1 activation and suppresses the glucose metabolism in macrophages. J Anesth, 24 (1), 54-60.

Taylor, E. B. (2017). Functional Properties of the Mitochondrial Carrier System. Trends Cell Biol, 27 (9), 633-644. doi:10.1016/j.tcb.2017.04.004

Veselkov, K. A., Lindon, J. C., Ebbels, T. M., Crockford, D., Volynkin, V. V., Holmes, E., . . Nicholson, J. K. (2009). Recursive segment-wise peak alignment of biological 1H NMR spectra for improved metabolic biomarker recovery. Analytical chemistry, 81 (1), 56-66.

Wright, E. M. (2020). SGLT2 and cancer. Pflugers Arch, 472 (9), 1407-1414. doi:10.1007/s00424-020-02448-4

Wu, Z. F., Lee, M. S., Wong, C. S., Lu, C. H., Huang, Y. S., Lin, K. T., . . . Lai, H. C. (2018). Propofolbased Total Intravenous Anesthesia Is Associated with Better Survival Than Desflurane Anesthesia in Colon Cancer Surgery. Anesthesiology, 129 (5), 932-941. doi:10.1097/aln.0000000000002357

Wyld, L., Audisio, R. A., \& Poston, G. J. (2015). The evolution of cancer surgery and future perspectives. Nat Rev Clin Oncol, 12 (2), 115-124. doi:10.1038/nrclinonc.2014.191

Xiao, F., Lv, J., Liang, Y. B., Chen, Y. H., Tu, Y. B., Guan, R. C., . . Xie, Y. B. (2020). The expression of glucose transporters and mitochondrial division and fusion proteins in rats exposed to hypoxic preconditioning to attenuate propofol neurotoxicity. Int J Neurosci, 130 (2), 161-169. doi:10.1080/00207454.2019.1667784

Xue, Y., Li, Z., Wang, Y., Zhu, X., Hu, R., \& Xu, W. (2020). Role of the HIF-1 $\alpha /$ SDF-1/CXCR4 signaling axis in accelerated fracture healing after craniocerebral injury. Mol Med Rep, 22 (4), 2767-2774. doi:10.3892/mmr.2020.11361

Yoo, H. C., Yu, Y. C., Sung, Y., \& Han, J. M. (2020). Glutamine reliance in cell metabolism. Exp Mol Med . doi:10.1038/s12276-020-00504-8

Zhang, F., Wang, C., Cui, Y., Li, S., Yao, Y., Ci, Y., . . . Li, E. (2016). Effects of Propofol on Several Membrane Characteristics of Cervical Cancer Cell Lines. Cell Physiol Biochem, 40 (1-2), 172-182. doi: $10.1159 / 000452535$

Zhao, H., Iwasaki, M., Yang, J., Savage, S., \& Ma, D. (2014). Hypoxia-inducible factor-1: a possible link between inhalational anesthetics and tumor progression? Acta Anaesthesiol Taiwan, 52 (2), 70-76. 
doi:10.1016/j.aat.2014.05.008

Zhu, Z.-J., Hu, Y., Zhao, Y.-F., Chen, X.-Z., Chen, L.-Q., \& Chen, Y.-T. (2011). Early Recurrence and Death After Esophagectomy in Patients With Esophageal Squamous Cell Carcinoma. The Annals of Thoracic Surgery, 91 (5), 1502-1508. doi:10.1016/j.athoracsur.2011.01.007

Zou, H., Chen, Q., Zhang, A., Wang, S., Wu, H., Yuan, Y., . . . Xu, C. (2019). MPC1 deficiency accelerates lung adenocarcinoma progression through the STAT3 pathway. Cell Death Dis, 10 (3), 148-148. doi:10.1038/s41419-019-1324-8

\section{Figure Legends}

Fig 1. The cell viability, proliferation, migration, and invasion of ovarian cancer cells after sevoflurane or propofol administration.

Ovarian cancer (SKOV3) cells were administered with pure culture media (naïve control), $2.5 \%$ sevoflurane, or $4 \mu \mathrm{g} / \mathrm{mL}$ propofol. The cell viability of ovarian cancer cells was evaluated with cell counting kit-8 assay (A). The cell proliferation of ovarian cancer cells was evaluated by identifying Ki-67 positive cells (B and C). The migration of SKOV3 cells was evaluated by calculating gap closure with wound healing assay (D and E). The invasion was evaluated by identifying invasive cells with Transwell assay ( $\mathrm{F}$ and $\mathrm{G})$. The data was expressed as mean \pm standard deviation and dots plot $(\mathrm{n}=6)$. ${ }^{*} \mathrm{p}<0.05,{ }^{* *} \mathrm{p}<0.01,{ }^{* * * *} \mathrm{p}<0.0001$ versus naïve control. Scale bar: $100 \mu \mathrm{m}$. NC, naïve control group; S, sevoflurane group; P, propofol group.

Fig 2. The effects of sevoflurane and propofol on the expressions of GLUT1, MPC1, and GLUD1 in ovarian cancer cells.

SKOV3 cells were exposed to media (naïve control), $2.5 \%$ sevoflurane, or $4 \mu \mathrm{g} / \mathrm{mL}$ propofol. The expressions of GLUT1 (A) and MPC1 (B) were detected with immunofluorescent staining. The expression levels of these two markers were validated with Western blot analysis and the expression level of GLUD1 was also analysed (C). The intensity of the bands in Western blot analysis was normalised by housekeeping protein GAPDH. The data was analysed with one-way ANOVA and Dunnett multi-comparison test (D-F) and was presented as mean \pm standard deviation and dots plot $(\mathrm{n}=6) .{ }^{*} \mathrm{p}<0.05,{ }^{* *} \mathrm{p}<0.01,{ }^{* * *} \mathrm{p}<0.001,{ }^{* * * *} \mathrm{p}<0.0001$ versus naïve control. Scale bar: $50 \mu \mathrm{m}$. NC: naïve control group; S: sevoflurane group; P: propofol group; GLUT1: glucose transporter 1; MPC1: mitochondrial pyruvate carrier 1; GLUD1: glutamate dehydrogenase 1; ANOVA: analysis of variance.

Fig 3. The metabolomics study of sevoflurane- or propofol-treated ovarian cancer cells using ${ }^{1}$ H-NMR spectroscopy.

Ovarian cancer cells were administered with media (naïve control), intralipid (vehicle control), $2.5 \%$ sevoflurane or $4 \mu \mathrm{g} / \mathrm{mL}$ propofol for 2 hours plus 24 hours recovery time. After treatment, the culture media of ovarian cancer SKOV3 cells was collected for ${ }^{1} \mathrm{H}-\mathrm{NMR}$ spectroscopy experiment. PCA scores plot of ${ }^{1} \mathrm{H}-\mathrm{NMR}$ spectra of media was shown (A). $\mathrm{R}^{2} \mathrm{X}$ represented the fraction of variance in the ${ }^{1} \mathrm{H}-\mathrm{NMR}$ data modelled by two principal components ( $\mathrm{t}[1]$ and $\mathrm{t}[2])$. The OPLS-DA scores plots (B: NC vs . S; D: VC vs . P) and loadings plots (C: NCvs. S; E: VC vs . P) were derived from ${ }^{1} \mathrm{H}-\mathrm{NMR}$ spectral data $(\mathrm{n}=10)$. The colour bar indicated the correlation coefficient values $\left(\mathrm{r}^{2}\right)$ to be high in red and low in blue. NC: naïve control group; VC: vehicle control group; S: sevoflurane group; P: propofol group; PCA: principal component analysis; OPLS-DA: orthogonal projection to latent structures-discriminant analysis; Glc: glucose; Lac: lactate; Pyr: pyruvate; Gln: glutamine; Ace: acetate; Ala: alanine; IPA: isopropanol; Val: valine; Leu: leucine; Eth: ethanol; Gro: glycerol; Asn: asparagine; FA: fatty acids; Suc: succinate; Acn: acetone; Arg: arginine; Ile: isoleucine.

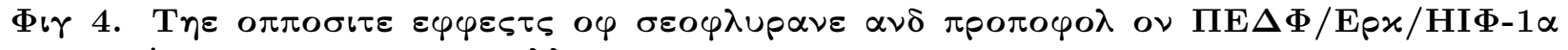

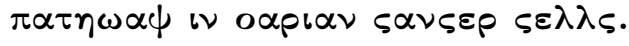

Ovarian cancer cells were administered with media (naïve control), $2.5 \%$ sevoflurane, or $4 \mu \mathrm{g} / \mathrm{mL}$ propofol. The expressions of PEDF (A) and HIF-1 $\alpha$ (B) were detected with immunofluorescence. The expression levels 
of PEDF, p-Erk1/2, Erk1/2, and HIF-1 $\alpha$ were evaluated with Western blot analysis (C). The intensity of PEDF and HIF- $1 \alpha$ bands was normalised by GAPDH (D and F). While the intensity of p-Erk1/2 band was normalised by Erk1/2 (E). Data was analysed with one-way ANOVA with Dunnett multi-comparison test and presented as mean \pm standard deviation and $\operatorname{dots}$ plot $(\mathrm{n}=6) .{ }^{*} \mathrm{p}<0.05,{ }^{* * * *} \mathrm{p}<0.0001$ versus naïve control. Scale bar: $50 \mu \mathrm{m}$. NC: naïve control group; S: sevoflurane group; P: propofol group; PEDF: pigment epithelium-derived factor; p-Erk1/2: phospho-extracellular-signal-regulated kinase 1 and 2; HIF-1 $\alpha$ : hypoxia-inducible factor-1 alpha; ANOVA: analysis of variance.

Fig 5. The expressions of CXCL12 and CXCR4 in ovarian cancer cells after sevoflurane or propofol exposure.

SKOV 3 cells were exposed to pure culture media (naïve control), $2.5 \%$ sevoflurane, or $4 \mu \mathrm{g} / \mathrm{mL}$ propofol for 2 hour and followed by 24 hours recovery. The expressions of CXCL12 (A) and CXCR4 (B) were detected with immunofluorescent staining (green) and overlaid with DAPI (blue). Sevoflurane exposure increased the expressions of CXCL12 and CXCR4 in ovarian cancer cells, while propofol decreased the expressions of both markers. NC: naïve control group; S: sevoflurane group; P: propofol group; CXCL12: C-X-C motif chemokine ligand 12; CXCR4: C-X-C motif chemokine receptor 4. 

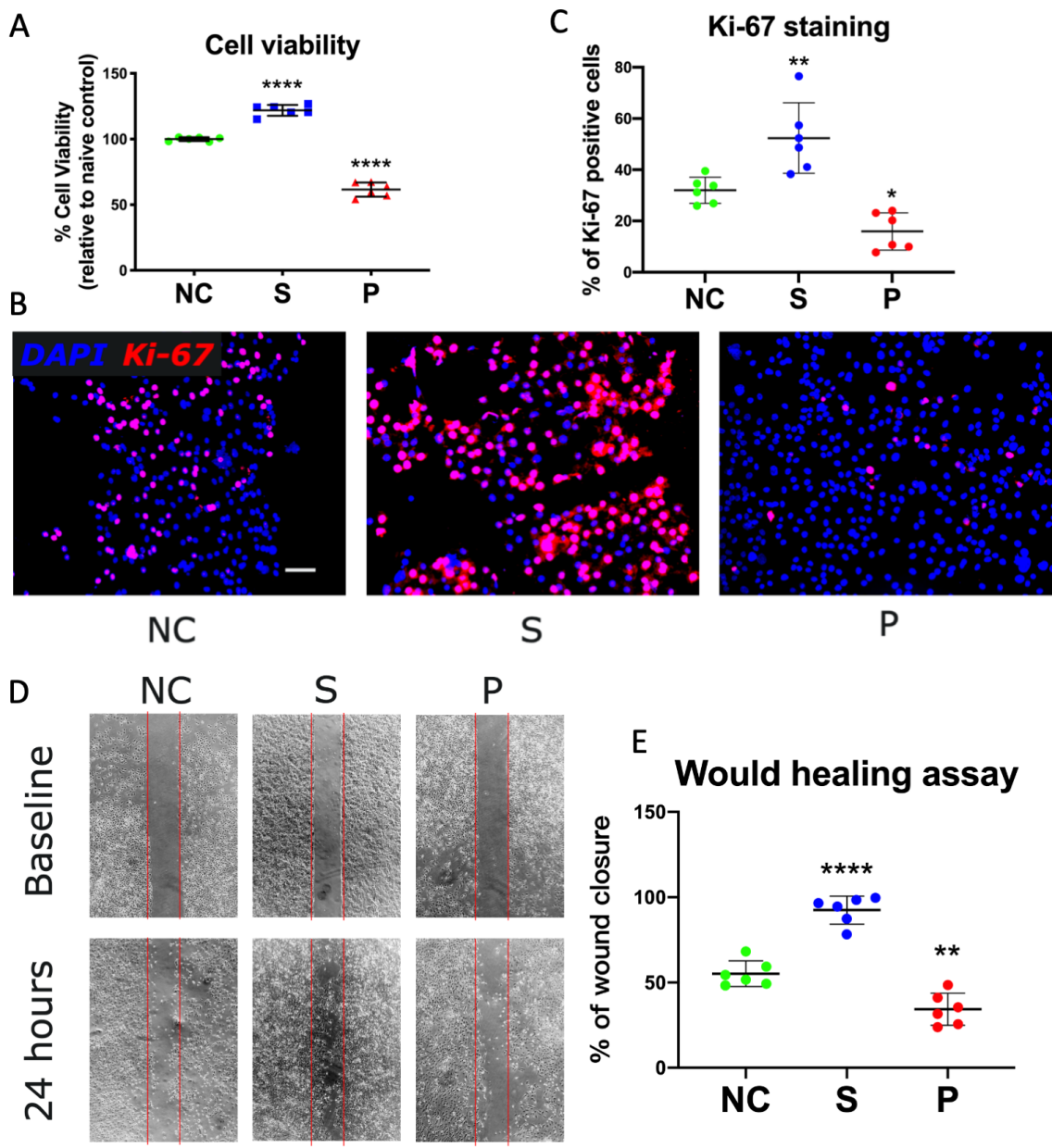

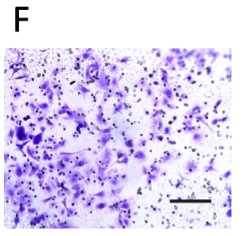

NC

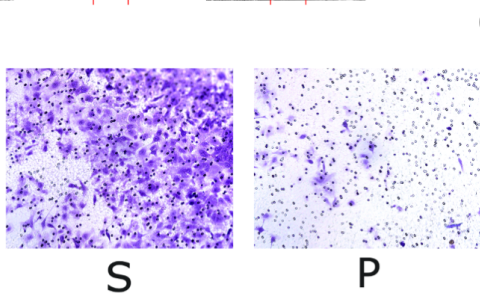

S
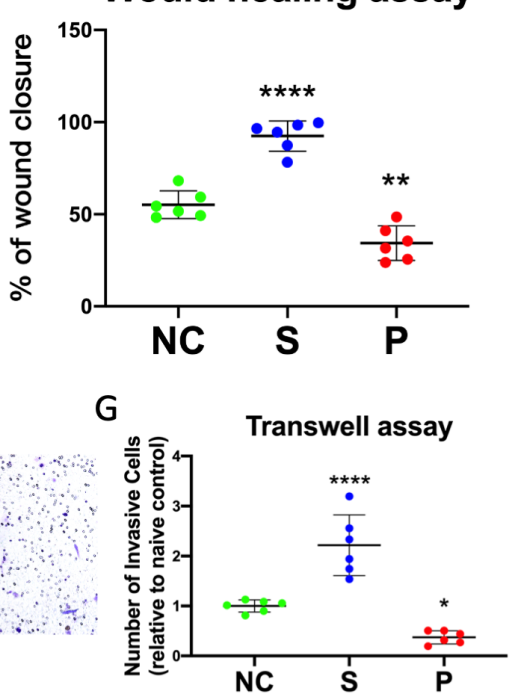

Figure 1 

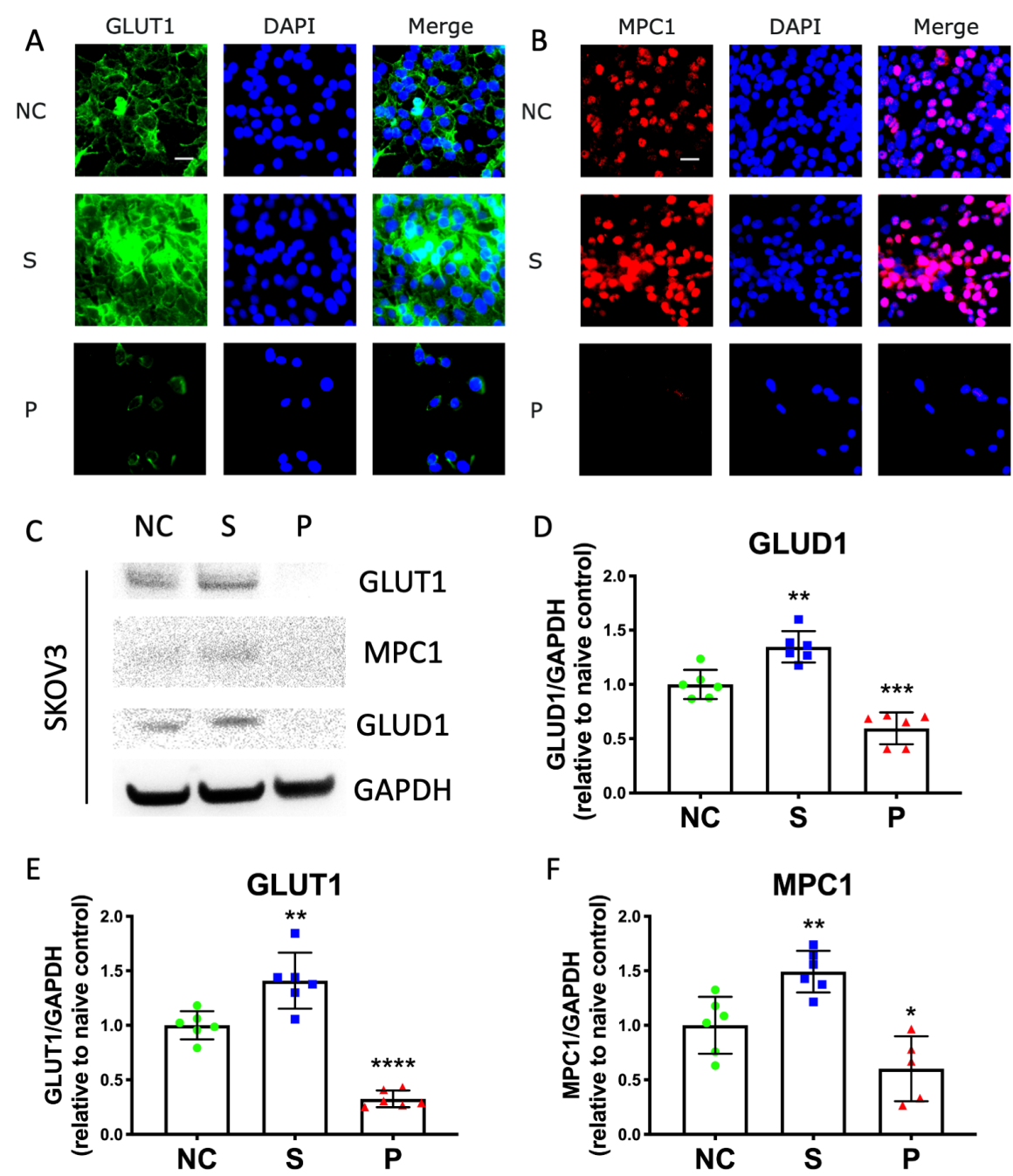

Figure 2 

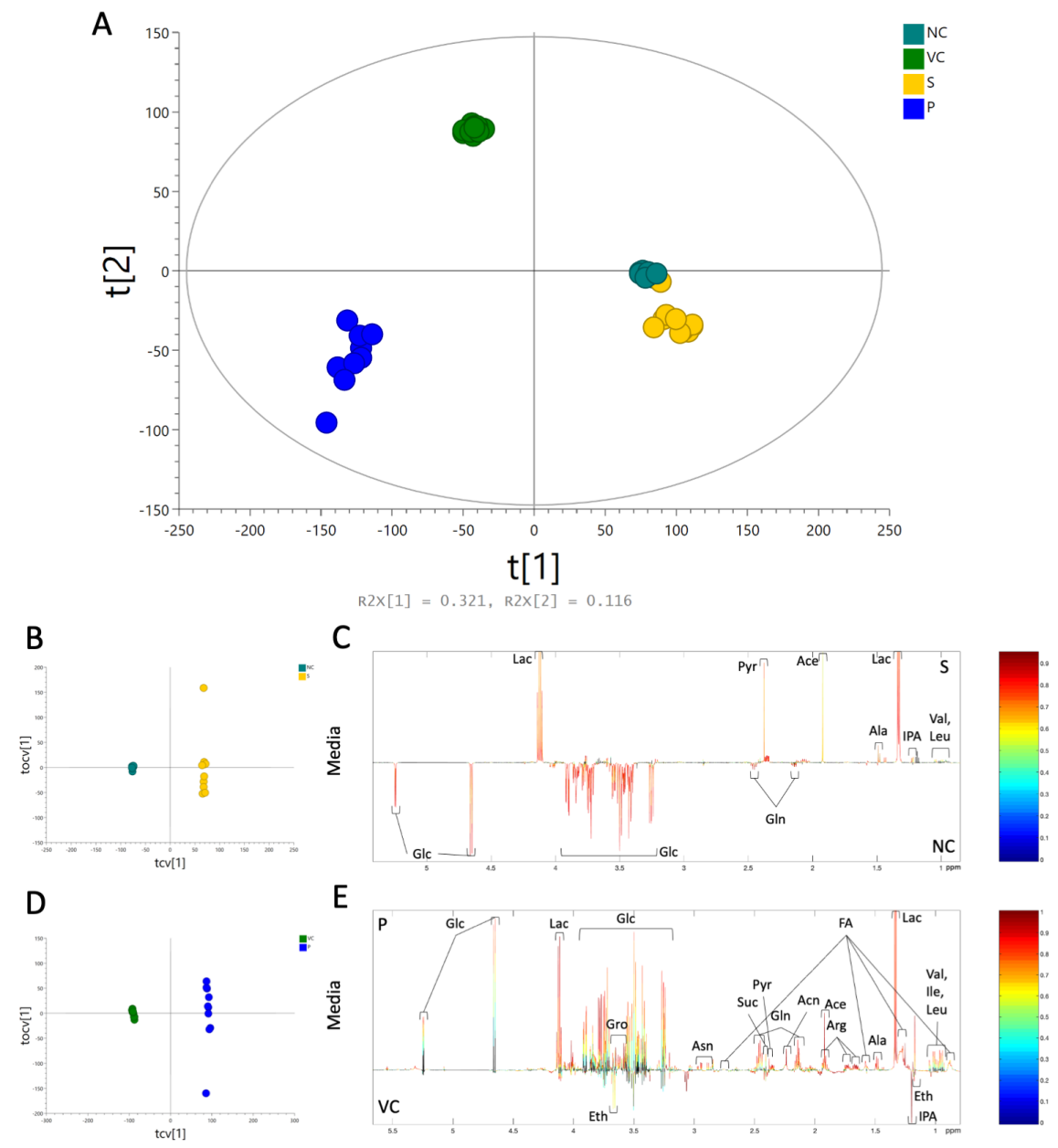

Figure 3 

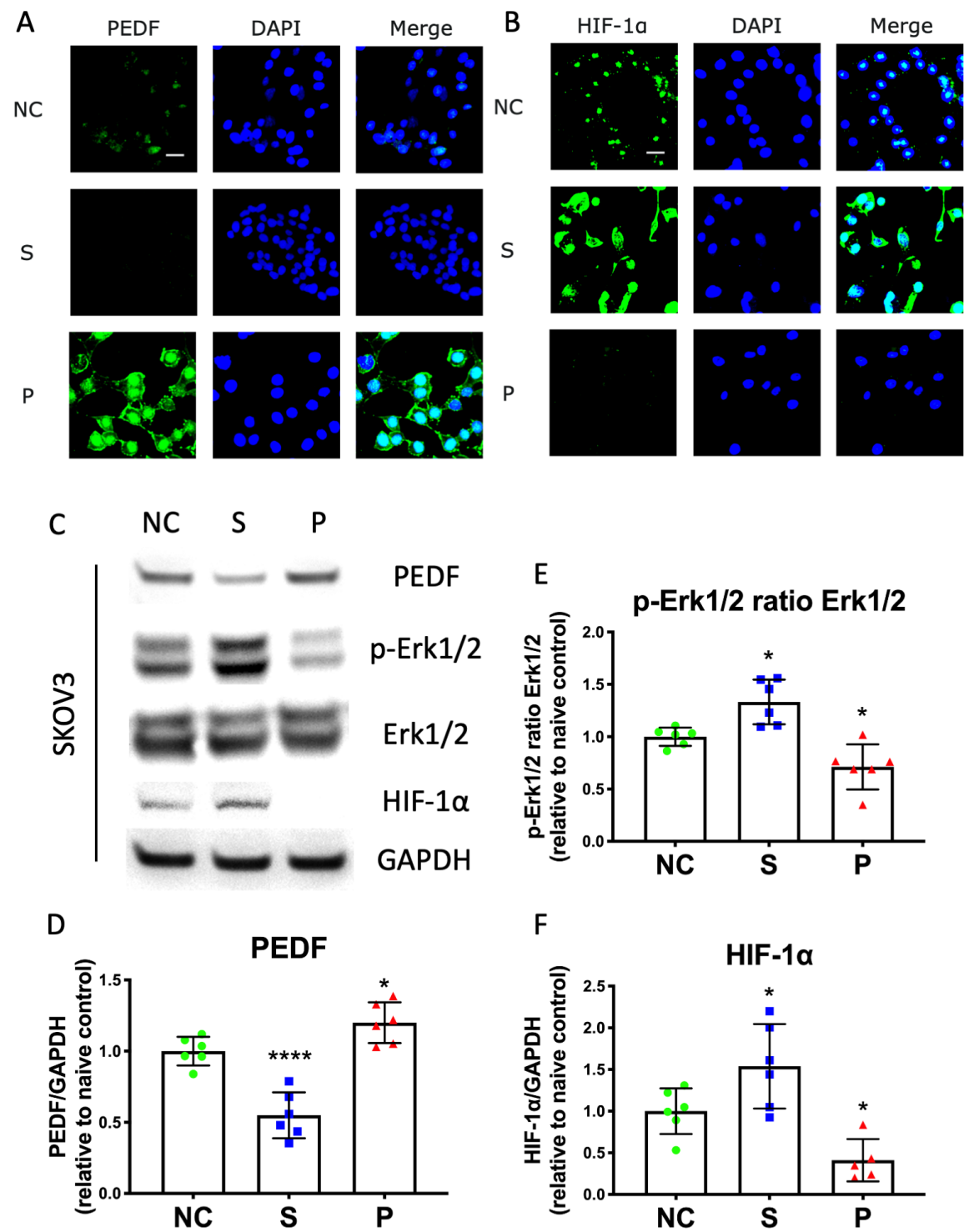

Figure 4 


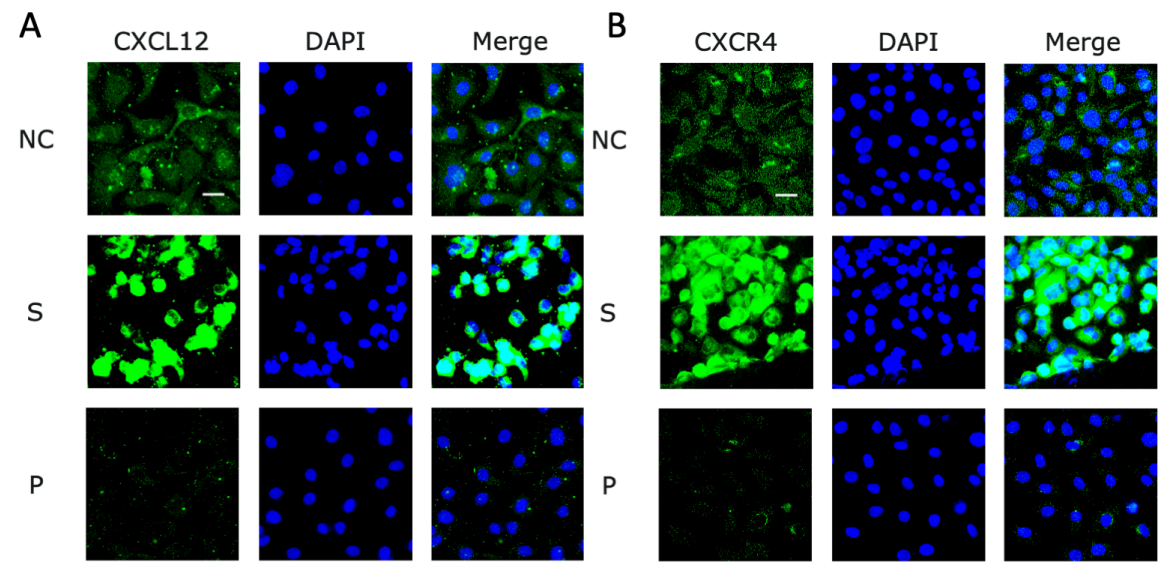

Figure 5

\section{Hosted file}

Tables.pdf available at https://authorea.com/users/398071/articles/510772-sevofluranebut-not-propofol-enhances-ovarian-cancer-cell-malignancy-through-regulating-cellularmetabolic-and-signalling-mechanisms 